\title{
Acercamiento a la morfología verbal del español guaymí costarricense $^{1}$
}

\section{(Approach to the Verbal Morphology of Costa Rican Guaymí Spanish)}

Rebeca Marín Esquive/2

Universidad Nacional, Costa Rica

\begin{abstract}
RESUMEN
Se describen y analizan las principales características de la morfología verbal del español hablado por tres indígenas del grupo étnico guaymí/ngäbe costarricense. Se transcribe ortográficamente el material oral obtenido en entrevistas con dos hablantes bilingües guaymí-español y uno monolingüe de español, hijo de guaymíes bilingües. En el marco de un estudio exploratorio, se concluye que las formas irregulares de los paradigmas analizados muestran indicios de regularización de las raíces que las asemeja más a su infinitivo, sumados a la creación de neologismos verbales.
\end{abstract}

\begin{abstract}
Key features of the verbal morphology of Spanish spoken by three indigenous speakers of the Costa Rican Guaymí/Ngäbe ethnic group are described and analyzed. Audio material was transcribed from interviews with two bilingual Guaymí-Spanish speakers and one Spanish monolingual son of bilingual Guaymí-Spanish parents. In this exploratory study, the irregular forms of the paradigms analyzed were found to show signs of regularizing the roots most closely resembling their infinitive, in addition to the creation of verbal neologisms.
\end{abstract}

1 Recibido: 3 de febrero de 2020, aceptado: 9 de junio de 2020.

2 Escuela de Literatura y Ciencias del Lenguaje. Correo electrónico: rebekamarin87@gmail.com

LETRAS 69 (2021), ISSN 1409-424X; EISSN 2215-4094 
Palabras clave: español, lenguas en contacto, guaymí, bilingüismo Keywords: Spanish, languages in contact, Guaymí, bilingualism

\section{Introducción}

La investigación sobre la influencia de las lenguas indoamericanas en el español de los hablantes indígenas ha ampliado el conocimiento sobre la diversidad lingüística hispanoamericana; es el caso de las variedades del español en contacto con el maya, el náhuatl, el quechua, el mapuche y el guaraní, documentadas en los estudios de Palacios (2008) y otros autores ${ }^{3}$. Esta variedad viene a revelar características morfosintácticas que incluyen simplificaciones en el sistema pronominal átono de objeto directo dada la neutralización del género a favor del masculino (pero yo no lo quería soltar a mi mamá) ${ }^{4}$, u omisiones del clítico (¿ahí ø [lo]compraste?) ${ }^{5}$, así como neutralizaciones de género y número entre componentes de la estructura oracional (esas mujeres que se habían quedado mudos) ${ }^{6}$. Además, es significativo que el influjo del nahuatl en los matices aspectuales del español en contacto con esta lengua, dada la tendencia al empleo de formas verbales progresivas por encima del presente simple (está queriendo mujer derivado de ki-nek-tok siwaatl: 3era objeto-querer progresivo 'mujer' en vez de quiere casarse, muchas de estas transmitidas intergeneracionalmente a la descendencia monolingüe (hijos y nietos de abuelos competentes en náhuatl).

Según Palacios (2011), los rasgos comunes entre esas variedades se originan en la convergencia de factores multicausales que involucran procesos de adquisición lingüística e influjo directo o indirecto de las

3 Azucena Palacios, El español en América. Contactos lingüisticos en Hispanoamérica (Barcelona: Ariel: 2008) 17-317.

4 Ana García, «Guatemala», El español en América. Contactos lingüisticos en Hispanoamérica (Barcelona: Ariel, 2008) 95-114.

5 Esmeralda de La Vega y Marleen Haboud, «Ecuador», El español en América. Contactos lingüisticos en Hispanoamérica (Barcelona: Ariel, 2008) 161-187.

6 Miguel Ángel Quesada, «América Central», El español en América. Contactos lingüisticos en Hispanoamérica (Barcelona: Ariel, 2008) 57-69. 
propiedades estructurales de las lenguas que entran en contacto con el español. Así, los bilingües pertenecientes a comunidades bilingües de lengua indígena (L1)-español (L2), han formado una interlengua en el proceso de adquisición de L2, la cual presenta rasgos particularizados que pueden llegar a fosilizarse en el español hablado por estos individuos al punto de formar un etnolecto. La lengua de contacto viene a funcionar como un factor lingüístico externo involucrado en las transferencias lingüísticas de una lengua a otra, así como en la reestructuración de patrones por influjo indirecto.

Las lenguas y variedades lingüísticas de Costa Rica han posibilitado la formación de un interesante abanico multilingüístico. Además de los (geo)lectos característicos de las regiones del centro, norte, noroeste, suroeste y pacífico-central; en las zonas del pacíficosureste y atlántico, se concentra una amplia diversidad de comunidades inmersas en situaciones de contacto con otras lenguas, tales como guatuso (malecu), cabécar, bribri, térraba (brorán), guaymí (ngäbere) y bocotá (buglere).

Centrándonos en la comunidad guaymí, su población se distribuye en cinco territorios ubicados en el sureste del país (algunos limitando con Panamá), estos son: el territorio de Abrojos Montezuma localizado en el Cantón de Corredores; el de Conte Burica, entre los Cantones de Corredores y Golfito; el de Coto Brus, entre los Cantones de Coto Brus y Buenos Aires; el de Alto Laguna de Osa, en el Cantón de Osa, Distrito, y el de Altos de San Antonio, en el Cantón de Corredores. Los guaymíes costarricenses forman parte de la diversidad lingüística de los territorios mencionados y de nuestro país en general. Se trata de una población mayoritariamente agrícola, aunque en los últimos años, los más jóvenes vienen accediendo a cargos de maestros de lengua, entre otros oficios.

Para esta comunidad, Marín ${ }^{7}$ documentó algunas características sobresalientes del español escrito por un guaymí costarricense como

7 Rebeca Marín, «Un acercamiento al español escrito por un guaymí costarricense», Escuela de Literatura y Ciencias del Lenguaje, 9 de enero de 2013, <www.literatura.una.ac.cr>. 
lo son las discordancias entre elementos de frase nominal; confusiones, elisiones y usos particulares de las preposiciones; además de discordancias entre verbo y sujeto. En 2017, esta misma autora indagó en los usos clíticos de tercera persona para objeto directo e indirecto en el registro oral de seis hablantes, obteniendo como resultado que, el empleo del clítico lo se encuentra por encima de la cantidad de omisiones de objeto directo y, dentro de las apariciones de $l o$, sus discordancias con los sintagmas correferenciales supera a los clíticos la(s) y los, lo cual refleja un proceso de simplificación del sistema pronominal átono de tercera persona ${ }^{8}$. Empero, dada la falta de más estudios que permitan tener un conocimiento más profundo de los fenómenos lingüísticos presentes en el español hablado por los indígenas guaymíes de Costa Rica, parece adecuado mostrar los apuntes recogidos sobre los rasgos lingüísticos de estas variedades de español, especialmente a nivel morfológico en el componente verbal.

\section{Generalidades morfosintácticas del guaymí como lengua de contacto}

La lengua guaymí, denominada como ngäbere (ngäbe) por sus hablantes, forma parte del grupo lingüístico chibcha ubicado en la Baja Centroamérica ${ }^{9}$. Entre las generalidades tipológicas ${ }^{10}$, el orden básico del guaymí es SOV, presenta posposiciones, la frase nominal generalmente se compone de adjetivo y sustantivo (en ese orden), la marcación de número se hace de manera opcional por medio del morfema -tre, y las palabras se forman mediante procesos de derivación, composición y reduplicación. Los verbos se clasifican en regulares (köt-, raíz de 'reir' para todos los TAM) e irregulares en distintas raíces (big-, raíz de 'intentar': bigue IND, jababa PT, etc.),

8 Rebeca Marín, «El sistema pronominal átono de tercera persona de objeto directo en el español hablado por guaymíes costarricenses», Revista de Filología y Lingüística 43, 2 (2017): 145-166.

9 Adolfo Constenla, Las lenguas del Área Intermedia: Introducción a su estudio areal (San José: EUCR, 1991).

10 José Manuel Murillo, Gramática de la lengua guaymí. Dialecto de Costa Rica: una descripción urgente (Saarbrücken: EAE, 2016). 
y su morfología se caracteriza por la presencia de afijos y auxiliares, entre otros elementos; además, se trata de una lengua que categoriza tiempo, aspecto/aktionsarten y modo, tal como se observa en el ejemplo, donde el verbo nigui 'ir' codifica la categoría temporal de pasado reciente de modo realis ${ }^{11}$ :

$$
\begin{aligned}
& \text { Nigui tre jökrä } \\
& \text { ir-REC PL todo } \\
& \text { 'se metieron todos'. }
\end{aligned}
$$

\section{El cambio inducido por contacto de lenguas}

En el ámbito de estas investigaciones surge, propiciado por distintos escenarios sociolingüísticos presentes en Hispanoamericana, el modelo del cambio lingüístico inducido por contacto, que se propone para explicar los rasgos de las variedades del español hablado en zonas multilingües ${ }^{12}$. Este modelo contempla que los hablantes inmersos en regiones bilingües y expuestos a diferentes lenguas y variedades suelen ser muy productivos en innovaciones lingüísticas de diversa índole. Los rasgos de las variedades de los hablantes bilingües se caracterizan por estas innovaciones, las cuales se extienden a los hablantes monolingües integrantes de esta misma comunidad multilingüe. El cambio lingüístico lo induce el contacto entre dos o más lenguas que se hablan en una misma región, por ejemplo, el español y una lengua indígena, y constituye un proceso que se dinamiza por el uso cotidiano de todas las lenguas implicadas; a su vez, los cambios que consolidan nuevas variedades de habla pueden estar restringidos a un idiolecto particular o extenderse a toda la comunidad (sistemática o asistemáticamente).

11 Miguel Ángel Quesada, Gramática de la lengua guaymí(ngäbe) (Barcelona: Ariel, 2008) 49-147.

12 Azucena Palacios, «Nuevas perspectivas en el estudio del cambio inducido por contacto: hacia un modelo dinámico del contacto de lenguas», Revista de Lenguas Modernas 38 (2011): 17-36. 
Según Palacios $(2011)^{13}$, los cambios inducidos por contacto de lenguas se originan en determinadas modalidades bilingües y se extienden hacia otras, inclusive monolingües. En este sentido, los límites entre las modalidades son lo suficientemente difusos como para que los rasgos lingüísticos característicos de las modalidades bilingües traspasen esos límites y aparezcan en las modalidades monolingües. El dinamismo con el que se extienden los rasgos ha hecho posible clasificar los cambios inducidos en dos tipos: cambios directos e indirectos. Según Palacios (2011), el primer tipo lo constituyen los cambios caracterizados por ocurrir asistemáticamente, casi siempre en hablantes bilingües consecutivos de lengua indígena como L1 (con mayor dominio de esta) o en bilingües simétricos L1 = L2 (con dominio aproximado), aunque pueden aparecer también en monolingües de una lengua u otra. En los cambios directos se identifica la introducción de sintagmas aislados y estructuras sintácticas provenientes de la lengua de mayor dominio para el hablante bilingüe, por ejemplo, Péa katu, atyra [un montón] de personas acudieron ayer a los locales de Amandau por el día nacional del helado gratis, en que se observa la trasferencia directa de léxico y estructuras gramaticales en quechua. Sin embargo, dentro de este tipo de cambios también se puede contemplar la incorporación de patrones sintácticos como Damos comprando, damos vendiendo... 'compramos, vendemos...', patrón de ruegos y peticiones basado en una relación análoga del quechua a la de 'dar' + gerundio en español.

Los cambios indirectos incluyen la reelaboración y la reorganización de elementos que forman parte de un sistema o subsistema de la lengua que está siendo influenciada por otra, y en la cual existe tendencia a la variación y al cambio. Ejemplo de ello se observa en el sistema de clíticos de tercera persona de objeto directo presente en el español hablado en zonas indígenas multilingües, en el cual se eliminan las restricciones de género y número con el fin de dar prioridad

13 Palacios (2011) 17-36. 
a la marcación de caso, mientras actúa el mecanismo de convergencia lingüística entre las estructuras de la lengua influyente (lengua indígena) que no presenta sistema de clíticos ni distinción de género y número gramatical, aunque sí distingue caso, y la lengua influida (español), cuyo sistema de clíticos distingue género, número y caso: Sí, chicha de jora lo hacemos (...) Esa chicha también lo preparan del maiz (ejemplo del español en contacto con el maya).

Esta tipología permite interpretar cada uno de los fenómenos presentes en el español hablado por guaymíes costarricenses como posibles cambios inducidos por contacto, ya sean directos o indirectos, dilucidando aquellas propiedades de la lengua guaymí que influyen en la conformación de estas variedades del español.

\section{Metodología}

El corpus toma como base un conjunto de materiales lingüísticos orales recolectados mediante entrevistas con tres maestros de primaria (varones) con bachillerato universitario concluido, dos de ellos bilingües español-guaymí, denominados HJ y HG de 36 y 32 años respectivamente, y uno monolingüe de español (ME), hijo de padres bilingües de español-guaymí, de treinta años y residente en el Territorio Indígena de Coto Brus. Se trata de una muestra pequeña debido al tiempo que tomó aplicar las entrevistas y a la poca disponibilidad de otros individuos para colaborar al momento de la investigación.

Estos hablantes proporcionaron textos orales variados, a saber, narraciones de eventos, acontecimientos personales, opiniones y deseos que fueron transcritos ortográficamente (entre una hora y una hora veinte la grabación de cada entrevistado), y se escogieron los textos cuya temática tiende a generar la producción de los mismos verbos en todos los hablantes ${ }^{14}$, de modo que fue un requisito en la organización del corpus seleccionar los verbos flexionados que compartieran una

14 Por ejemplo, en un relato sobre la muerte, los hablantes suelen producir diferentes flexiones del verbo 'morir'. 
misma forma básica, por ejemplo, 'quería'(forma flexionada) de 'querer' (forma básica). Se clasificaron los rasgos morfológicos observables en los verbos; $y$, en tercer lugar, se recogió material lingüístico oral de un hispanocostarricense de treinta y tres años (HC), universitario y residente de un sector aledaño, con el propósito de observar si determinados fenómenos podrían tener algún condicionamiento dialectal más que etnolectal ${ }^{15}$. En este bosquejo de naturaleza exploratoria, no se pretende llegar a conclusiones finales, ni mucho menos exactas de los hallazgos identificados en los corpus; por el momento, tan solo se procuran explicar a la luz del referente teórico de la lingüística del contacto ${ }^{16}$.

\section{Descripción y análisis de los datos lingüísticos}

La mayoría de las formas verbales de la muestra sigue las reglas de formación de palabras del español ${ }^{17}$; se mantienen las raíces, la secuencia de los sufijos de flexión, entre los cuales se puede mencionar la vocal temática, el TAM (tiempo, aspecto y modo) y la desinencia verbal, por ejemplo, estaba, confió y dijo; asimismo, se evidencian

15 Quesada (2008) documenta rasgos de la morfología verbal del español (sin contacto) hablado en Costa Rica desde el siglo xviii hasta inicios del siglo xxi. A inicios del siglo xvii, se empleaban las terminaciones verbales -ades y -des presente en segunda persona plural (hazed execusión de los bienes que hallardes), mientras que la primera persona singular de indicativo (lo cual reprodusgo) $\mathrm{y}$ todas las personas del presente subjuntivo (redusgan a sus pueblos) alternaron -cer con -zc- y -sg- hasta el siglo xix. Desde la colonia, son muy comunes las alternancias en los verbos como 'traer' (trajo $\sim$ trujo) 'producir' (produjo $\sim$ produció) y 'ver' (veía $\sim$ vía, vide $\sim$ vi y vido $\sim$ vio) con tendencia a la monoptongación (traer $\sim$ trer), las regularizaciones en tercera persona plural del pretérito perfecto simple de indicativo a favor de -ieron (traxieron) y de -iera $\sim$-iese (trujiese) de los verbos traer y decir. Además, el futuro de poder, tener y haber restituye la $e$ de la marca verbal (poderá, tenerá y haberá), tendencia muy propia de los manuscritos del siglo xvii. Las formas de infinitivo (dixeron ser naturales del pueblo de Pacaca.) y el gerundio (abiendo disipado) estas se documentan en manuscritos de la época colonial hasta el siglo xix con la misma morfología del español costarricense actual. Según el autor, aún en el siglo xxi se escuchan todas estas formas en zonas rurales del país, aunque no especifica en cuáles exactamente y en qué condiciones sociolingüísticas, etnográficas, etc.

16 Por su naturaleza exploratoria, se considera prematuro centrar el análisis en la cuantificación de tendencias de uso de las formas verbales, de ahí que se haya preferido verificar únicamente presencia o ausencia en determinados hablantes.

17 Real Academia de la Lengua Española (RAE), Manual de la nueva gramática de la lengua española (Madrid: Asociación de Academias de la Lengua Española, 2010). 
también formas compuestas como están jugando, había ido y podían consumir. Sin embargo, en el conjunto de los datos se observan fenómenos alejados del panorama descrito, los cuales se organizan aquí en dos conjuntos: verbos irregulares y regulares. A continuación se describen los rasgos morfológicos de los verbos identificados en cada hablante. Para cada apartado, se hizo un cuadro comparativo en el que se aprecian las formas de tiempo, aspecto y modo (TAM) ${ }^{18}$ identificadas durante el proceso de análisis.

\section{Verbos irregulares}

Del universo de verbos irregulares emitidos por los entrevistados HJ, HG, ME y HC, se identificó modificaciones en las raíces de querer, morir, poder y encontrar. Las flexiones de cada una de estas formas verbales se aprecian en los cuadros del 1 al 4 :

Cuadro 1. Flexiones registradas de 'querer'

\begin{tabular}{|c|c|c|c|c|c|c|c|}
\hline Verbo & TAM & PN & Forma & HJ & HG & ME & $\mathrm{HC}$ \\
\hline \multirow{5}{*}{ querer } & $\begin{array}{l}\text { Presente } \\
\text { indicativo }\end{array}$ & $\begin{array}{l}\text { 1era } \\
\text { Sing. }\end{array}$ & quiero & Sí & Sí & Sí & Sí \\
\hline & $\begin{array}{l}\text { Presente } \\
\text { indicativo }\end{array}$ & $\begin{array}{l}2 \text { era } \\
\text { Sing. }\end{array}$ & quiere & Sí & Sí & Sí & Sí \\
\hline & $\begin{array}{l}\text { Pret. imp. } \\
\text { indicativo }\end{array}$ & $\begin{array}{l}\text { era } \\
\text { PL. }\end{array}$ & querían & Sí & Sí & Sí & Sí \\
\hline & \multirow{2}{*}{$\begin{array}{l}\text { Pret. imp. } \\
\text { subjuntivo }\end{array}$} & \multirow{2}{*}{$\begin{array}{l}3 \text { era } \\
\text { Sing. }\end{array}$} & queriera & Sí & Sí & No & No \\
\hline & & & quisiera & No & Sí & Sí & Sí \\
\hline
\end{tabular}

HJ y HG flexionan el pretérito imperfecto de subjuntivo en tercera persona singular del verbo querer, como queriera (1), mientras que ME y HC lo flexionan como quisiera (2):

... él buscó a una mujer porque él esperaba de esa mujer que queriera a los dos niños, pero no fue así...

18 La forma verbal producida por el hablante generalmente aparece flexionada; sin embargo, para efectos de exposición, en la primera columna de los cuadros se añade la forma infinitiva. 
(2) ... [ella] quisiera viajar a San José a meter papele al ministerio...

El presente indicativo de segunda y primera persona aparece flexionado en HJ, HG, ME y HC como 'quiere' (3) y 'quiero' (4) respectivamente:

(3) ... me enojé porque uté no quiere casarse conmigo...

(4) ... quiero ver a esa señora...

En cuanto al pretérito imperfecto de indicativo de querer, su flexión en tercera persona plural se registra como querían (5) para los cuatro hablantes:

... querían a la señora como abuela...

Cuadro 2. Flexiones registradas de 'morir'

\begin{tabular}{|c|c|c|c|c|c|c|c|}
\hline Verbo & TAM & PN & Forma & HJ & HG & ME & HC \\
\hline \multirow{7}{*}{ Morir } & \multirow{2}{*}{$\begin{array}{l}\text { Pret. simp. } \\
\text { indicativo }\end{array}$} & \multirow{2}{*}{\begin{tabular}{|l} 
3era \\
PL.
\end{tabular}} & morieron & Sí & Sí & No & No \\
\hline & & & murieron & Sí & Sí & Sí & Sí \\
\hline & \multirow{2}{*}{$\begin{array}{l}\text { Pret. simp. } \\
\text { Indicativo }\end{array}$} & \multirow{2}{*}{\begin{tabular}{|l|} 
3era \\
Sing.
\end{tabular}} & morió & Sí & Sí & Sí & No \\
\hline & & & murió & Sí & Sí & Sí & Sí \\
\hline & $\begin{array}{l}\text { Pret. imp. } \\
\text { indicativo }\end{array}$ & \begin{tabular}{|l|} 
era \\
Sing.
\end{tabular} & moría & Sí & Sí & Sí & Sí \\
\hline & \multirow[t]{2}{*}{ Gerundio } & \multirow[t]{2}{*}{-} & moriendo & Sí & No & No & No \\
\hline & & & muriendo & No & Sí & Sí & Sí \\
\hline
\end{tabular}

El pretérito simple de indicativo se flexiona en tercera persona plural como morieron en HJ y HG (6) y como murieron (7) en los cuatro entrevistados:

(6) ... la dos persona se morieron en la montaña...

(7) ... mis abuelos murieron hace años... 
HJ, HG y ME flexionan el pretérito simple de indicativo en tercera persona singular como morió (8), aunque también se registra como murió (9) en las muestras orales de los tres guaymíes y HC:

(8) ... se morió ahogado en la poza, no había nadie...

(9) ... murió mi mamá, y después, murió mi papá...

La forma de pretérito imperfecto de indicativo en tercera persona singular se flexiona como moría (10) para HJ, HG, ME y HC:

(10) ... la gente se moría de hambre...

Además, la forma de gerundio se registra en $\mathrm{HJ}$ como moriendo (11), mientras que en HG, ME y HC se registra como muriendo (12):

(11) ... estaba moriendo... moriendo... de enfermedad porque ya no quedaba cura...

(12) ... fue muriendo [mi abuelo] lentamente... no pudimos hacer casi nada...

Cuadro 3. Flexiones registradas de 'poder'

\begin{tabular}{|c|c|c|c|c|c|c|c|}
\hline Verbo & TAM & PN & Forma & HJ & HG & ME & HC \\
\hline \multirow{6}{*}{ poder } & \multirow{2}{*}{$\begin{array}{l}\text { Pret. imp. } \\
\text { subjuntivo }\end{array}$} & \multirow{2}{*}{$\begin{array}{l}\text { 3era } \\
\text { Sing. }\end{array}$} & podiera & Sí & No & No & No \\
\hline & & & pudiera & No & Sí & Sí & Sí \\
\hline & $\begin{array}{l}\text { Pret. imp. } \\
\text { indicativo }\end{array}$ & $\begin{array}{l}3 \text { era } \\
\text { Sing. }\end{array}$ & podía & Sí & Sí & Sí & Sí \\
\hline & $\begin{array}{l}\text { Pret. imp. } \\
\text { indicativo }\end{array}$ & $\begin{array}{l}\text { era } \\
\text { PL. }\end{array}$ & podían & Sí & Sí & Sí & Sí \\
\hline & \begin{tabular}{|l|} 
Presente \\
indicativo
\end{tabular} & $\begin{array}{l}\text { lera } \\
\text { PL. }\end{array}$ & podemos & Sí & Sí & Sí & Sí \\
\hline & $\begin{array}{l}\text { Presente } \\
\text { indicativo }\end{array}$ & $\begin{array}{l}\text { 1era } \\
\text { Sing. }\end{array}$ & puedo & Sí & Sí & Sí & Sí \\
\hline
\end{tabular}


La forma podiera, de pretérito imperfecto de subjuntivo, se registra únicamente en el hablante $\mathrm{HJ}$ (13), mientras que su variante pudiera se identifica en HG, ME y HC (14):

(13) ... si la muchacha podiera llevar el agua ahí...

(14) ... cuando el niño pudiera o estuviera listo tenía que entrar en la jaula...

Otras flexiones de pretérito y presente aparecen en el habla de $\mathrm{HJ}, \mathrm{HG}, \mathrm{ME}$ y HC: pretérito imperfecto de indicativo singular en tercera persona podía (15), pretérito imperfecto de indicativo plural en tercera persona podían (16), presente en primera persona plural podemos (17) y presente de indicativo en primera persona puedo (18):

(15) ... la señora no podía sentarse sola...

(16) ... si lo chiquillo podían volver, debía engañarlos de nuevo...

(17) ... podemos llevar las cosas tranquilos...

(18) ... es mi beca, puedo utilizar a lo que yo quiera ...

Cuadro 4. Flexiones registradas de 'encontrar'

\begin{tabular}{|c|l|l|l|l|l|l|l|}
\hline Verbo & \multicolumn{1}{|c|}{ TAM } & \multicolumn{1}{|c|}{ PN } & \multicolumn{1}{|c|}{ Forma } & \multicolumn{1}{|c|}{ HJ } & \multicolumn{1}{|c|}{ HG } & \multicolumn{1}{|c|}{ ME } & \multicolumn{1}{c|}{ HC } \\
\hline \multirow{4}{*}{ Encontrar } & $\begin{array}{l}\text { Pret. imp. } \\
\text { indicativo }\end{array}$ & $\begin{array}{l}\text { 3era } \\
\text { PL. }\end{array}$ & encontrabas & Sí & No & No & No \\
\cline { 2 - 8 } & $\begin{array}{l}\text { Presente } \\
\text { indicativo }\end{array}$ & $\begin{array}{l}\text { 3era } \\
\text { Sing. }\end{array}$ & encuentraban & Sí & Sí & Sí & Sí \\
\hline
\end{tabular}

En HJ, se presenta la forma de pretérito imperfecto de indicativo en tercera persona plural como encontrabas (19), mientras que en $\mathrm{HG}$ y ME aparece como encontraban (20):

(19) ... Empezaron [ellos] [a] buscar comida porque ya no habia opción... comieron [ellos] las frutas que encontrabas, las hojas que podían consumir...

(20) cuando los papás llegaban, encontraban muchos problemas... 
Con respecto al presente indicativo de tercera persona encuentra (21), este se registra en el material oral de los tres guaymíes HJ, HG y ME y del hispanocostarricense $\mathrm{HC}$ :

(21) ... encuentra [el niño] una señora ciega cocinando... Observaciones sobre los verbos irregulares

En los cuadros 1 y 3 , las raíces verbales de querer y poder del pretérito imperfecto de subjuntivo en tercera persona, se flexionan para HJ únicamente como queriera y podiera en analogía con la forma básica de infinitivo; en cambio, en HG, si bien es cierto también coexisten las variantes queriera y quisiera, dicho subjuntivo de poder solo se realiza como pudiera. Con respecto a ME, quisiera es la única forma registrada para este TAM.

En el cuadro 2, la raíz de morir, del pretérito simple de indicativo en tercera persona, se flexiona como morieron y murieron. Ambas formas se registran tanto para $\mathrm{HJ}$ como para $\mathrm{HG}$, mientras que para ME, se identifica solamente el empleo de murieron entre las dos posibilidades registradas. Por su parte, este mismo pretérito flexionado en tercera persona singular, se presenta en los hablantes HJ, HG y ME como morió y murió, lo que hace evidente la coexistencia entre ambas formas sumada a una posible analogía con el morfema radical de infinitivo morir. Asimismo, el gerundio registrado como moriendo se aparece únicamente en el hablante $\mathrm{HJ}$ sin mostrar coexistencia alguna con muriendo, mientras que, en los hablantes HG y ME, muriendo corresponde a la única forma identificada en las muestras orales de ambos hablantes.

En el cuadro 4, el pretérito imperfecto de tercera persona plural de encontrar se registra como encontrabas y encontraban. Desde esta perspectiva, el morfema codificador de número y persona $-s$ que en el español sin contacto se utiliza para marcar segunda persona ${ }^{19}$, aquí

19 Miguel Ángel Quesada, Historia de la lengua española en Costa Rica. (San José: Editorial de Costa Rica, 2009) 308-340. 
se utiliza para codificar la tercera persona plural, posiblemente por analogía con el morfema $-s$ de los sustantivos en plural.

Se deben destacar tres aspectos en torno a los verbos irregulares. Primero: HJ produce flexiones de querer, morir y poder utilizando la raíz básica del infinitivo: queriera y querían; morieron, morió, moría y moriendo, y podiera podía, podían y podemos; sin embargo, en este entrevistado, dichas flexiones no se presentan de modo sistemático en relación con otras entre diferentes tiempos y personas verbales, puesto que también se identifican flexiones irregulares como quiere y quiero, murieron, murió y puedo. Segundo: en HG se presentan todas las flexiones registradas para querer, lo cual implica que, para el presente simple de subjuntivo en tercera persona, coexisten las formas irregular quisiera y la regularizada queriera. No obstante, para morir y poder, este tipo de coexistencias no parecen ser sistemáticas, por cuanto para $\mathrm{HG}$ se registra la coexistencia de morieron y murieron, aunque no de moriendo y muriendo, ni de podiera y pudiera. Tercero: en ME tan solo se identifica regularización de la raíz de pretérito simple de indicativo en tercera persona morió y murió, donde ambas formas coexisten. $\mathrm{HC}$, por su parte, utiliza las flexiones verbales documentadas para el español estándar ${ }^{20}$.

\section{Verbos regulares}

HJ, HG, ME y HC producen las flexiones para los verbos lamer y comprometer que se observan en cuadros 5 y 6 :

Cuadro 5. Flexiones registradas de 'lamer'

\begin{tabular}{|c|c|c|c|c|c|c|c|}
\hline Verbo & TAM & PN & Forma & HJ & HG & ME & HC \\
\hline \multirow{4}{*}{ lamer } & \multirow[t]{2}{*}{ Infinitivo } & \multirow[t]{2}{*}{ - } & lamiar & Sí & Sí & No & No \\
\hline & & & lamer & No & No & Sí & Sí \\
\hline & \multirow{2}{*}{$\begin{array}{l}\text { Pret. imp. } \\
\text { indicativo }\end{array}$} & \multirow{2}{*}{$\begin{array}{l}\text { 3era } \\
\text { Sing. }\end{array}$} & lamiaba & Sí & No & No & No \\
\hline & & & lamía & No & Sí & Sí & Sí \\
\hline
\end{tabular}

20 RAE. 
Los entrevistados $\mathrm{HJyHG}$ producenla forma infinitivaúnicamente como lamiar (22), en tanto ME y HC la producen como lamer (23):

(22) ... y empezó a lamiar con la lengua en parte del cuerpo, en el pecho ...

(23) ... la historia dice, según me contaron, que el perro empezó como a chupar o a lamer...

La flexión lamiaba para el pretérito imperfecto de indicativo solo se registra en $\mathrm{HJ}$ (24), mientras en HG, ME y HC se presenta como lamía (25):

(24) ... si el perro lamiaba fuerte, y mucho mucho, podía resucitarlo...

(25) ... el animal se lamía mucho, bastante, como acicalándose diría uno...

Cuadro 6. Flexiones registradas de 'comprometer'

\begin{tabular}{|c|l|l|l|l|l|l|l|}
\hline Verbo & TAM & \multicolumn{1}{c|}{ PN } & \multicolumn{1}{c|}{ Forma } & \multicolumn{1}{c|}{ HJ } & \multicolumn{1}{c|}{ HG } & \multicolumn{1}{c|}{ ME } & \multicolumn{1}{c|}{ HC } \\
\hline Comprometer & $\begin{array}{l}\text { Pret. } \\
\text { simp. } \\
\text { ind. }\end{array}$ & $\begin{array}{l}\text { 3era } \\
\text { Sing. }\end{array}$ & competió & Sí & No & No & No \\
\cline { 2 - 8 } & & & Nomprometió & Sí & Sí & Sí \\
\hline
\end{tabular}

El pretérito simple de indicativo en tercera persona singular se registra en HJ como competió (26) y en HG, ME y HC como 'comprometió' (27).

(26) ... había suquía oeste o norte o sur que [se] competió a volver para regresar la lluvia...

(27) ... iSí, claro! Se comprometió a tenerlos ahí, pero al final no lo hizo... 
Observaciones sobre los verbos regulares

En el cuadro 5 está el empleo de la vocal temática diptongada en el infinitivo de lamiar. Si bien es cierto, también se ha identificado la presencia de lamer en el corpus, no se registra coexistencia alguna entre ambas formas verbales para un mismo hablante. Algo similar ocurre con las flexiones de pretérito imperfecto de indicativo en tercera persona singular lamiaba y lamía, de las cuales la primera se produce únicamente en $\mathrm{HJ}$, mientras que la segunda solo se identifica en las hablas de $\mathrm{HG}, \mathrm{ME}$ y $\mathrm{HC}$, sin que para un mismo hablante se registren ambas formas. En todo caso, al menos lamiar puede ser considerada como neologismo verbal, dada su creación a partir de una analogía con los verbos de primera conjugación terminados en-(i)ar (repudiar, rociar, odiar, etc.).

En el cuadro 6 se muestra que el pretérito simple de indicativo aparece con dos tipos de raíces: una raíz irregularizada en competió, la cual ocurre solo en HJ, y otra comprometió, que conserva la forma básica de comprometer(se). La raíz de competió es el resultado de una síncopa o supresión de los sonidos [rom], posiblemente en un intento del hablante por facilitar la pronunciación de esa palabra en específico.

$\mathrm{Al}$ igual que en los verbos irregulares, las flexiones empleadas por HC corresponden a las documentadas para la variedad del español estándar ${ }^{21}$.

\section{Conclusiones}

Los medios de formación, flexión y organización de las formas verbales y sus paradigmas son bastante estables en los idiolectos $\mathrm{HJ}$, HG y ME, en el sentido de que la mayoría de las flexiones registradas coincide con el paradigma verbal documentado para el español estándar ${ }^{22}$. De todas maneras, resulta llamativa la regularización en los verbos querer, poder y algunos casos de morir. Esa regularización

21 RAE.

22 RAE. 
constituye en un mecanismo para simplificar el paradigma verbal, lo cual podría ser sintomático de un proceso de cambio indirecto (inducido por contacto) que toma como base las posibilidades estructurales sujetas a variación, estas son, las raíces quer- y quis- (de querer) y pod- y pud- (de poder) del pretérito imperfecto de subjuntivo de tercera persona, además de mur- y mor- (de morir) identificadas en el gerundio y el pretérito simple de indicativo de tercera persona ${ }^{23}$.

Con respecto a los verbos regulares, los rasgos recolectados sobre las formas de lamer permiten postular tentativamente un sistema más vernacular representado en HJ (lamiar, lamiaba), un sistema transicional en HG (lamiar, lamía) y un sistema plenamente estándar en ME (lamer, lamía), el cual es igual al sistema de HC. Si bien las diferencias de edades son pocas (HJ, 36 años; $\mathrm{HG}, 32$; ME, 30 y HC, $33)$, la asociación entre las edades y el grado de vernacularización parece ser tentadora.

Para finalizar, es recomendable que en futuros estudios se cuantifique la tendencia de cada hablante guaymí (bilingüe o monolingüe) a escoger determinadas posibilidades estructurales disponibles en el paradigma verbal, ya sean estas más básicas (conservando la raíz del infinitivo) o menos básica (prefiriendo la raíz irregular) en comparación, así como aumentar la muestra poblacional e indagar más a profundidad en las biografías lingüísticas de los hablantes.

23 Pese a que el guaymí como lengua de contacto posee verbos regulares e irregulares, no es posible indicar que su influencia en el español de los entrevistados guaymíes se deba a un cambio directo, pues se trata de paradigmas verbales muy distintos entre los cuales no se observa transferencia de elementos o patrones. 


\section{Abreviaturas}

IND. Indefinido

Ind. Indicativo

Inf. Infinitivo

Imp. Imperfecto

M Modo

O Objeto

PL. Plural

Pret. Pretérito

PT. Pasado testimonial

REC. Pasado reciente

Sing. Singular

Simp. Simple

$\mathrm{S}$

T Tiempo

V Verbo 